\title{
Improved library preparation protocols for amplicon sequencing-based noninvasive fetal genotyping for RHD-positive D antigen-negative alleles
}

\author{
Asuka Hori ${ }^{1,2}$, Hiroko Ogata-Kawata ${ }^{1}$, Aiko Sasaki ${ }^{3}$, Ken Takahashi $^{4}$, Kosuke Taniguchi $^{1}$, Ohsuke Migita ${ }^{1,5}$, \\ Akihiro Kawashima ${ }^{6}$, Aikou Okamoto ${ }^{4}$, Akihiko Sekizawa ${ }^{6}$, Haruhiko Sago ${ }^{3}$, Fumio Takada², \\ Kazuhiko Nakabayashi ${ }^{7^{*+}}$ (10 and Kenichiro Hata ${ }^{{ }^{*+}}$
}

\begin{abstract}
Objective: We aimed to simplify our fetal RHD genotyping protocol by changing the method to attach Illumina's sequencing adaptors to PCR products from the ligation-based method to a PCR-based method, and to improve its reliability and robustness by introducing unique molecular indexes, which allow us to count the numbers of DNA fragments used as PCR templates and to minimize the effects of PCR and sequencing errors.

Results: Both of the newly established protocols reduced time and cost compared with our conventional protocol. Removal of PCR duplicates using UMIs reduced the frequencies of erroneously mapped sequences reads likely generated by PCR and sequencing errors. The modified protocols will help us facilitate implementing fetal RHD genotyping for East Asian populations into clinical practice.
\end{abstract}

Keywords: RHD, Cell-free DNA (cfDNA), Non-invasive prenatal testing (NIPT), Amplicon sequencing, Unique molecular identifier (UMI)

\section{Introduction}

Alloantibodies against $\mathrm{Rh}$ antigens represent the main cause of hemolytic disease of the fetus and newborn (HDFN). The D antigen is the most highly immunogenic among $\mathrm{Rh}$ antigens. $\mathrm{RhD}$-negative women become sensitized to the $\mathrm{D}$ antigen and subsequently produce

*Correspondence: nakabaya-k@ncchd.go.jp; hata-k@ncchd.go.jp ${ }^{\dagger}$ Kazuhiko Nakabayashi and Kenichiro Hata contributed equally to this work

1 Department of Maternal-Fetal Biology, National Research Institute for Child Health and Development, 2-10-1 Okura, Setagaya,

Tokyo 157-8535, Japan

${ }^{7}$ Laboratory of Developmental Genomics, National Research

Institute for Child Health and Development, 2-10-1 Okura, Setagaya,

Tokyo 157-8535, Japan

Full list of author information is available at the end of the article
anti-D antibodies when they carry an RhD-positive fetus. Although anti-D prophylaxis by postnatal and antenatal anti-D Ig administration has been highly successful in reducing the incidence of HDFN worldwide [1], it is unnecessary for RhD-negative women who carry an RhD-negative fetus. Fetal RHD genotyping makes it possible to prevent unnecessary anti-D administration in such pregnancy cases. The fetal RHD genotyping method widely implemented in western countries is designed to detect the presence or absence of the $R H D$ wild-type allele of fetal origin in the plasma of RhD-negative pregnant women, over $99.9 \%$ of whom are homozygous for $R H D$ deletion alleles in Caucasian populations. Because of relatively high frequencies of $R H D$-positive RhD-negative alleles, RHD*01EL.01 and RHD*01N.04, 


\begin{abstract}
(See figure on next page.)
Fig. 1 Conventional and newly developed library preparation protocols for amplicon sequencing-based noninvasive fetal RHD genotyping. A Genomic organization of the RHD/RHCE locus. Open and closed boxes indicate upstream and downstream Rhesus boxes. Closed and open arrowheads indicate PCR primer pairs to amplify 105-bp intervals in Rhesus boxes and 148-bp intervals spanning the exon 9 and the intron 9 of RHD and RHCE genes, respectively. Amplicon-seq libraries without UMI can be prepared by our conventional ligation-based protocol (B, C) and the one-step multiplex PCR protocol (E, F) established in this study. Amplicon-seq libraries with UMI can be prepared by the newly established linear and PCR amplification protocol $(\mathbf{H}, \mathbf{I})$. Approximate total and hands-on time $(\mathbf{B}, \mathbf{E}, \mathbf{H})$, diagrams for experimental procedures $(\mathbf{C}, \mathbf{F}, \mathbf{I})$, and representative electropherograms of intermediate PCR products and/or final libraries ( $\mathbf{D}, \mathbf{G}, \mathbf{J})$ are shown for three protocols. In the panels $\mathbf{C}, \mathbf{F}$, and $\mathbf{I}$, genomic DNA is shown by a black line; PCR primers that target genomic DNA sequences are shown by blue arrows; adaptor sequences are shown in dark/light green/orange; 12-base UMIs are shown in red. Index sequences to de-multiplex sequence reads after sequencing of pooled libraries are omitted for simplicity. Panels $\mathbf{D}, \mathbf{G}$, and $\mathbf{J}$ show representative electropherograms of Rhesus box amplicons ("box"), for RHD/RHCE exon 9 amplicons ("ex9"), and for multiplex amplification by two pairs of primers ("multiplex"). Horizontal and vertical axes of each electropherogram represent fluorescent intensity and DNA size (bp), respectively. Lower and upper marker peaks are present at the positions of $35 \mathrm{bp}$ and 10,380 bp, respectively. In our conventional [4] and one-step PCR protocols (C, F), multiplex PCR using two pairs of primers gave rise to specific amplification of target genomic intervals $(\mathbf{D}, \mathbf{G})$. K A data analysis workflow showing the informatics tools used and their step-by-step functions. $\mathbf{L}$ Bam coverage tracks of the RHD genotyping results by the one-step PCR protocol (F) for three combinations of the mixtures of genomic DNA (combinations $\# 1$, \#2 and \#3 in Additional file 2: Table S2) and for two cfDNA samples (\#46 and \#59) from RhD-negative pregnant women (Table 1) visualized using IGV. The mapped read patterns indicated that the RHD genotypes of the mother (Mo) and the fetus (Fe) were RHD*01N.01/RHD*01N.01 and $R H D^{*} 01 / R H D^{*} 01 N .01$, respectively, in both cases. The vertical ranges of mapped read numbers were $0-25,000$ for the top three panels, $0-40,000$ for cfDNA\#46, and 0-150,000 for cfDNA\#59
\end{abstract}

among RhD-negative individuals (9.0\% and 2.9\%, respectively, in the Japanese population), the same genotyping method was not applicable in East Asian countries. The RHD*01EL.01 allele contains a single nucleotide variant at the last nucleotide of exon 9 (c.1227G/A), which likely disrupts normal splicing [2]. The RHD*01N.04 allele is a hybrid allele, in which exons 3-9 of the RHD gene are replaced with those of $R H C E$ [3].

We have recently established an amplicon-based noninvasive fetal genotyping method that distinguishes the wild-type $R H D$ allele not only from the $R H D$-negative $\mathrm{D}$ antigen-negative allele (the $R H D$ deletion allele), but also from $R H D$-positive D antigen-negative alleles [4]. This method requires PCR amplification from four genomic intervals, upstream and downstream Rhesus boxes, RHD exon 9, and RHCE exon 9. Because of extremely high sequence similarities between two Rhesus boxes and between RHD exon 9 and RHCE exon 9, we designed two primer pairs to amplify these four regions (Fig. 1A, Additional file 1: Fig. S1). One primer pair perfectly matches with two genomic intervals, upstream and downstream Rhesus boxes, and amplifies 105-bp PCR products. The other primer pair also perfectly matches with two genomic intervals, $R H D$ exon 9 and $R H C E$ exon 9 regions, and amplifies 148-bp PCR products. The 105bp PCR products contain one base difference that distinguishes two Rhesus box sequences. The 148-bp PCR products contain two base differences that distinguish two genes, and also cover the point mutation site in exon 9 of the RHD*01EL.01 allele (c.1227G/A) that distinguishes it from the wild-type allele $\left(R H D^{*} 01\right)$. Although two regions are co-amplified with one primer pair, attachment of adaptor sequences to the PCR amplicons followed by NGS allowed us to accurately map each of the co-amplified sequences to its origin because of the one or two base differences between two regions, in the data analysis procedure [4].

In this study, we simplified our fetal $R H D$ genotyping protocol by changing the adaptor attachment method from ligation (Fig. 1B, C) to a one-step PCR (Fig. 1E, F). We also evaluated whether introduction of unique molecular indexes (UMIs) [5, 6] (Fig. 1H, I) improves the quantitative accuracy in measuring the ratios of $R H D$ alleles in cfDNA.

\section{Main text \\ Material and methods \\ Blood collection and DNA extraction}

Cell-free DNA in maternal plasma was extracted using the Mag MAX Cell-Free DNA Isolation Kit (ThermoFisher Scientific, A29319) as described previously [4]. Individuals with three major RhD-negative genotypes, RHD*01N.01/RHD*01N.01, RHD*01N.01/RHD*01.04, and $R H D * 01 N .01 / R H D * 01 E L .01$ in the Japanese population, and those with two $\mathrm{RhD}$-positive genotypes, $R H D^{*} 01 / R H D^{*} 01$ and $R H D^{*} 01 / R H D^{*} 01 N$.01, were identified as described previously [4].

\section{Preparation of amplicon sequencing libraries by one-step PCR (Fig. 1E, F)}

A tailed-forward primer ("Tailed_F") contains the Illumina forward (P5) adaptor sequence (70 bases) including an 8-bp index followed by a target-specific forward primer sequence ( 20 or 22 bases) at the $3^{\prime}$ end ( 90 or 92 bases in total). A tailed-reverse primer ("Tailed_R") contains the Illumina reverse (P7) adaptor sequence (66 


\section{A}

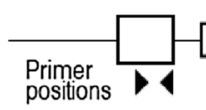

B

PCR and adaptor ligation (no UMI)

1. PCR amplification (x30) $90 \mathrm{~min}$

2. AMPure beads purification $40 \mathrm{~min}$

3. Bioanalyzer

4. DNA end repairs

5. Adaptor ligation

$70 \mathrm{~min}$

6. AMPure beads purification $40 \mathrm{~min}$

7. PCR amplification (x6) $45 \mathrm{~min}$

8. AMPure beads purification $40 \mathrm{~min}$

\begin{tabular}{ll} 
9. Bioanalyzer & $70 \mathrm{~min}$ \\
\hline Time total & $8.5 \mathrm{~h}$
\end{tabular}

Hands-on time total

$4.0 \mathrm{~h}$

C

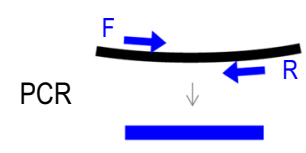

Adaptor ligation

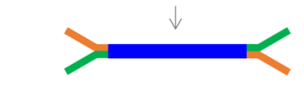

PCR

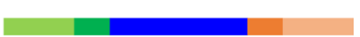

D

Step 3

Step 9

box
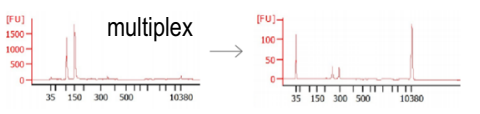

$\mathbf{K}$

fastg files (paired-end 150bp x2)

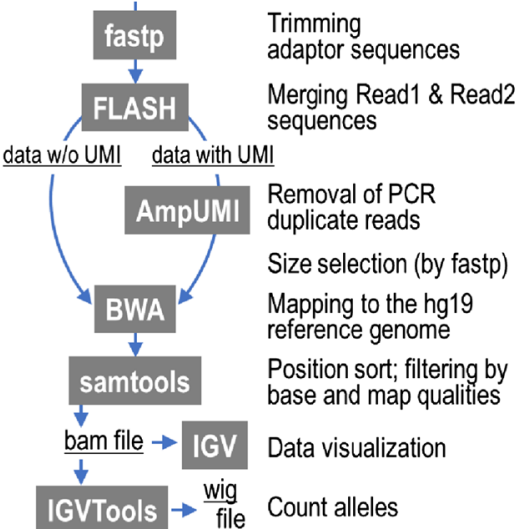

E

One-Step multiplex PCR (no UMI)

1. PCR amplification (x30) $90 \mathrm{~min}$

2. AMPure beads purification $40 \mathrm{~min}$

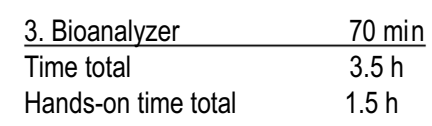

F

G
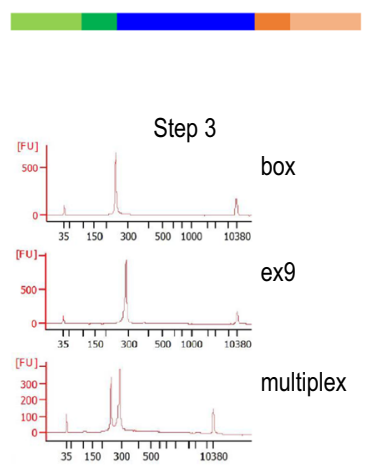

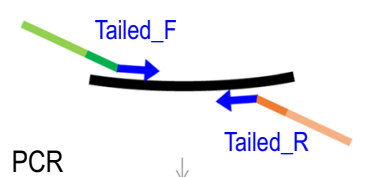

RHCE

H

Linear\&PCR amplification (with UMI)

1. Linear amplification $70 \mathrm{~min}$

2. PCR amplification (x35) $90 \mathrm{~min}$

3. AMPure beads purification $40 \mathrm{~min}$

4. AMPure beads purification $40 \mathrm{~min}$

5. Bioanalyzer $\quad 70 \mathrm{~min}$

Time total $\quad 5.5 \mathrm{~h}$

Hand-on time total $\quad 2.5 \mathrm{~h}$

I Linear 5, Tailed_F1_UMI12

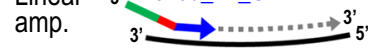

PCR 5' $3^{\prime} 4 \ldots \ldots \ldots \ldots \leftarrow 3^{\prime}$

Tailed_F2 $\quad \downarrow \quad$ Tailed_R $\quad$ 5'

5 '

J
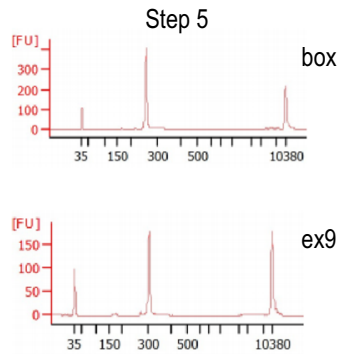

L
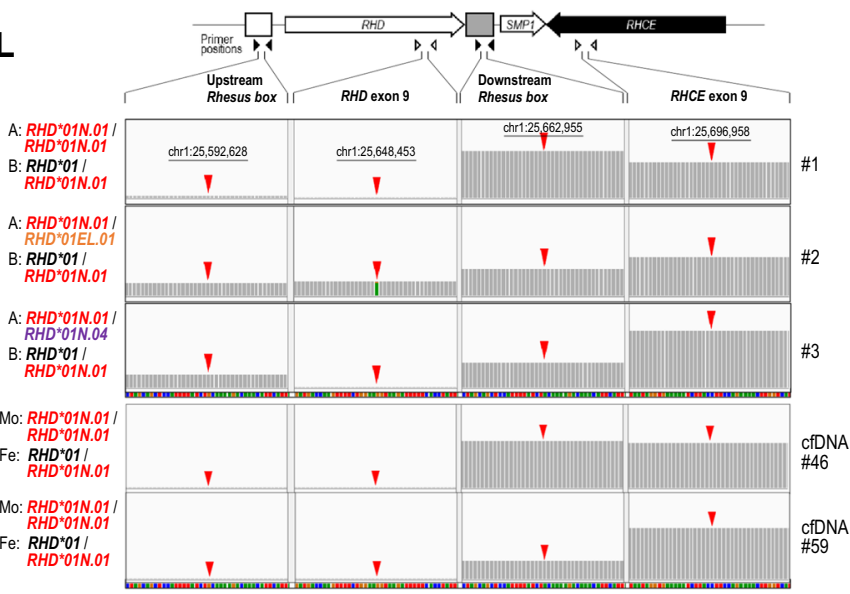

Fig. 1 (See legend on previous page.) 
bases) including an 8-bp index followed by a target specific reverse primer sequence ( 25 or 29 bases) at the $3^{\prime}$ end (Additional file 2: Table S1). PCR was performed with $2 \mathrm{ng}$ of a mixture of genomic DNA of two individuals (or cfDNA) using 0.5 unit of Q5 Hot Start High-Fidelity DNA Polymerase (M0493, NEB) according to the manufacturer's instruction in a $25 \mu \mathrm{L}$ reaction at the following final concentrations: $1 \times \mathrm{Q} 5$ reaction buffer, $0.2 \mathrm{mM}$ dNTPs, $0.5 \mu \mathrm{M}$ each of the tailed primers. The thermal cycling conditions used were $98{ }^{\circ} \mathrm{C}$ for $30 \mathrm{~s}, 30$ cycles of $98{ }^{\circ} \mathrm{C}$ for $10 \mathrm{~s}, 64{ }^{\circ} \mathrm{C}$ for $30 \mathrm{~s}$, and $72{ }^{\circ} \mathrm{C}$ for $30 \mathrm{~s}$, and $72{ }^{\circ} \mathrm{C}$ for $2 \mathrm{~min}$. Twenty out of the $25 \mu \mathrm{L}$ reactions were purified using 0.9 times the volume $(18 \mu \mathrm{L})$ of Agencourt AMPure XP (A63881, Beckman Coulter) and eluted with $10 \mu \mathrm{L}$ distilled water. The purified PCR products, i.e., the final libraries, were electrophoresed using the High Sensitivity DNA Kit on a 2100 BioAnalyzer (Agilent) to confirm their sizes, and subjected to paired-end sequencing $(151 \mathrm{bp} \times 2)$ on a MiSeq system (Illumina) using MiSeq Reagent Kit v2 Nano.

\section{Preparation of amplicon sequencing libraries with unique molecular identifier (UMI) sequences (Fig. 1H, I)}

Cell-free DNA was concentrated using a centrifugal evaporator (MicroVac MV-100, TOMY) when necessary. Linear amplification was performed in a $23 \mu \mathrm{L}$ scale reaction consisting of $8 \mathrm{ng}$ of a mixture of genomic DNA of two individuals in $10.35 \mu \mathrm{L}, 1.15 \mu \mathrm{L}$ of $0.4 \mu \mathrm{M}$ "Tailed_ F1_UMI12" primer (final concentration of 20 pM, Additional file 2: Table S1), and $11.5 \mu \mathrm{L}$ of Q5 Hot Start HiFi PCR Master Mix (M0543, NEB). The thermal conditions for linear amplification were $98{ }^{\circ} \mathrm{C}$ for $2 \mathrm{~min}, 57{ }^{\circ} \mathrm{C}$ for $15 \mathrm{~min}, 61^{\circ} \mathrm{C}$ for $15 \mathrm{~min}$, and $65{ }^{\circ} \mathrm{C}$ for $5 \mathrm{~min}$. Subsequently, $1.0 \mu \mathrm{L}$ each of $10 \mu \mathrm{M}$ "Tailed_F2" and "Tailed_R" primers, and $25.0 \mu \mathrm{L}$ of Q5 Hot Start HiFi PCR Master Mix were added to the $23 \mu \mathrm{L}$ reaction and mixed by pipetting. The resultant $50 \mu \mathrm{L}$ reactions were subjected to PCR amplification with the following conditions for Rhesus boxes: $98^{\circ} \mathrm{C}$ for $1 \mathrm{~min}$; 35 cycles of $98^{\circ} \mathrm{C}$ for $10 \mathrm{~s}$, $64{ }^{\circ} \mathrm{C}$ for $30 \mathrm{~s}$, and $68^{\circ} \mathrm{C}$ for $45 \mathrm{~s} ; 68^{\circ} \mathrm{C}$ for $5 \mathrm{~min}$, and for RHD/RHCE exon 9: $98{ }^{\circ} \mathrm{C}$ for $1 \mathrm{~min}$; 35 cycles of $98{ }^{\circ} \mathrm{C}$ for $10 \mathrm{~s}, 66^{\circ} \mathrm{C}$ for $30 \mathrm{~s}$, and $70{ }^{\circ} \mathrm{C}$ for $45 \mathrm{~s} ; 70{ }^{\circ} \mathrm{C}$ for $5 \mathrm{~min}$. Twenty out of the $50 \mu \mathrm{L}$ reactions were purified using 0.9 times the volume $(18 \mu \mathrm{L})$ of Agencourt AMPure XP repeated twice, and eluted with $10 \mu \mathrm{L}$ distilled water. The final libraries were electrophoresed and sequenced as described above.

\section{Data analysis (Fig. 1K)}

For amplicon libraries without UMIs, fastq files were generated using bcl2fastq V2.20.0.422 (Illumina), and trimmed for adaptor sequences using fastp ver.0.21.0 [7]. Read 1 and read 2 sequences were merged using
FLASH ver.1.2.11 [8] with a parameter of "-max-mismatch-density $=0$ ", and the merged sequences were filtered by their expected size (105 bases for Rhesus boxes and 148 bases for RHD/RHCE exon 9) to remove reads with unexpected sizes (such as primer dimers and PCR artefacts). The merged sequences were mapped to the hg19 reference genome using "bwa aln" and "bwa samse" commands of bwa-0.7.17 [9]. By using samtools -1.4.1 [10], the resultant bam file was sorted in a positional order and filtered by base quality scores (cutoff $25)$ to remove low quality reads, by mapping scores (cutoff 23 for Rhesus boxes and 37 for RHD/RHCE exon 9) to select uniquely mapped reads. The mapped read numbers were counted for each of the four bases at each nucleotide position of the amplicons using IGVTools_2.3.94 (https://software.broadinstitute.org/ software/igv/igvtools) (igvtools count-w 1-bases), and output as a.wig file. Subsequently, the numbers of $G$ at $\operatorname{chr} 1: 25,592,628$ and of $\mathrm{A}$ at $\operatorname{chr} 1: 25,662,955$ were extracted as read counts of the upstream and the downstream Rhesus boxes, respectively. The numbers of $\mathrm{G}$ and $A$ at chr1:25,648,453 as the read counts of the wildtype allele and the $1227 \mathrm{G}>\mathrm{A}$ allele of $R H D$ exon 9, and the number of $C$ at chr1:25,696,958 as the read count of $R H C E$ exon 9 were extracted. The mapping results were further examined for the existence of unexpected variants or potential sequence errors by visualizing the bam file data for four regions corresponding to the amplicons using IGV (https://software.broadinstitute. org/software/igv/), and by inspecting the minor allele frequency of each nucleotide position of the amplicons using the text data (.wig file) generated by IGVTools. For amplicon libraries with UMIs, fastq generation, adaptor trimming, and merge of paired reads were performed as described above. The resultant merged sequences were further processed with AmpUMI.py 1.2 [6] to remove PCR duplicate reads using 12-base UMI sequences located at the beginning of the original read 1. The sequence reads after removing PCR duplicates were further processed as described above.

The.wig file data were used to calculate the ratio of the read number containing the bases other than the reference base to the total read number at a single nucleotide (i.e., error ratio). These read numbers were initially counted for each nucleotide of the four amplicon regions (upstream and downstream Rhesus boxes, RHD exon 9, and RHCE exon 9). Error ratios were subsequently calculated using the total numbers for the positionally identical bases between the upstream and downstream at Rhesus box amplicons (for 105 positions) and between RHD exon 9 and RHCE exon 9 amplicons (for 147 positions excluding the position of the c.1227A $>\mathrm{G}$ variation at chr1: 25,648,453). 
Table 1 Mapped read ratios of one-step PCR amplicons from cell-free DNA of RhD-negative pregnant women

\begin{tabular}{lll}
\hline Cell-free DNA from a RhD-negative pregnant woman & $\begin{array}{l}\text { Amplicons from Rhesus boxes } \\
\text { (Primers: RHbox_Tailed_F/R) }\end{array}$ & $\begin{array}{l}\text { Amplicons from } R H D / R H C E \text { exon } \\
\text { 9 (Primers: RHD/RHCE_exon9_ } \\
\text { Tailed_F/R) }\end{array}$ \\
& $\begin{array}{l}\text { Observed ratio and read counts } \\
\text { Observed ratio and read counts } \\
\text { (RHD_wt/RHD_var/RHCE) }\end{array}$ \\
\hline Cell-free DNA \#46 (8 weeks) & $1.88 \% / 98.12 \%(416 / 21,694)$ & $1.33 \% / 0 \% / 98.67 \%(281 / 0 / 20,914)$ \\
Cell-free DNA \#59 (28 weeks) & $3.60 \% / 96.40 \%(813 / 21,754)$ & $\begin{array}{l}2.05 \% / 0.002 \% / 97.95 \% \\
(1,253 / 1 / 59,758)\end{array}$ \\
& &
\end{tabular}

The gestational week of blood sampling is shown in parentheses

\section{Results and discussion}

We established one-step PCR conditions (Fig. 1E-G) and tested on twelve combinations of genomic DNA mixtures of two individuals (A and $\mathrm{B}$ ) at a 10:1 ratio, which served as approximation models of cfDNA from RhD-negative pregnant women. "A" corresponds to the mother and is any of three RhD-negative genotypes (RHD*01N.01/ RHD*01N.01, RHD*01N.01/RHD*01EL.01, or RHD*01N.01/RHD*01N.04), and "B" corresponds to the fetus and is any of four genotypes (one RhD-positive genotype [RHD*01/RHD*01N.01] or three RhD-negative genotypes). These twelve combinations cover $93.6 \%$ of possible genotype combinations of a fetus and an RhDnegative pregnant woman in the Japanese population. The observed ratios of amplicons from Rhesus boxes and from $R H D / R H C E$ exon 9 were mostly consistent with the expected ratios (Additional file 2: Table S2). The reliability for estimating the fetal $\mathrm{RhD}$ type of the newly established one-step PCR protocol was confirmed to be comparable with that of the conventional protocol (Fig. 1B-D) [4]. Examples of mapped read data visualized by IGV are provided (Fig. 1L).

UMIs have been used to confidently detect PCR duplicates in NGS applications [6], and have been shown to reduce sequencing error rates and to increase analytical specificity in various studies, including those for NIPT $[11,12]$. We established linear and subsequent exponential amplification conditions to introduce 12-base UMIs to the amplicon libraries (Fig. $1 \mathrm{H}-\mathrm{J}$ ), and tested the protocol on the twelve combinations of genomic DNA mixtures described above (Additional file 2: Table S3). The results we obtained by the UMI protocol with our standard filtering conditions were almost completely free of erroneous reads, and were not suitable to assess the efficacy of UMI to reduce errors. When we analyzed the same dataset with less stringent filtering conditions, we confirmed that the UMI-based removal of PCR duplicates lowered the ratio of erroneous reads for the majority of cases (data not shown). We observed a stronger tendency of the amplification bias towards RHCE exon 9 over RHD exon 9 in the dataset by the linear \& PCR amplification protocol (Additional file 2:
Table S3) than that by one-step PCR (Additional file 2: Table S2). Further optimization of the linear and the PCR amplification conditions is required to minimize the observed amplification biases.

PCR and sequencing errors are inherent in the current NGS technologies [13]. We calculated the ratios of such errors presumably generated during library preparation and sequencing procedures and retained after applying read filtering conditions in the data analysis procedure. When the mapped read data of twelve samples in Additional file 2: Table S2 were analyzed, the highest error ratios were $0.06 \%$ and $0.11 \%$ for $R$ hesus box amplicons and RHD/RHCE exon 9 amplicons, respectively (Additional file 1: Fig. S2). Although potential PCR or sequencing errors at the nucleotide positions to distinguish the origin of each sequence read were observed at low frequencies, as shown in red in Table 1, Additional file 2: Tables S2 and S3, their ratios were below the calculated background levels except for one case $(0.19 \%)$ detected for the fourth combination of genomic DNA mixture (Additional file 2: Table S2). Such a high error ratio indicates the possibility of carry-over contamination from previous PCR assays. UMI is expected to be useful as a means to remove such contaminated reads.

In NIPT methods, fetal fraction (FF), the ratio of fetal DNA in cfDNA in maternal plasma, has been recognized as the most critical factor for their diagnostic accuracy [14]. The sensitivity of trisomy 21 detection dropped from 99 to $75 \%$ when FF was below 8\% [15]. In our method, when the $R H D^{*} 01$ allele is detected in cfDNA from maternal plasma, it determinately demonstrates that the fetus is RhD-positive. On the other hand, when the $R H D^{*} 01$ allele is undetected, it leaves two possibilities, namely, that the fetus is RhD-negative and homozygous for the RHD deletion (RHD*01N.01) allele, or that the assay failed to detect an RhDpositive allele of the fetus due to a low FF. Accurate determination of the target molecule number in a genotyping PCR reaction through UMI-based removal of PCR duplicates helps us better presume which one of the possibilities is more likely. 


\section{Limitations}

The limitation of our amplicon-sequencing-based fetal RHD genotyping is that the method by itself cannot determine FF, which is common to all amplicon-based NIPT methods. To complement this limitation, we plan to adopt an amplicon-sequencing method for multiple SNPs [16] when FF of a cfDNA sample needs to be precisely determined.

\author{
Abbreviations \\ cfDNA: Cell-free DNA; HDFN: Hemolytic disease of the fetus and newborn; \\ PCR: Polymerase chain reaction; NGS: Next generation sequencing; UMI: \\ Unique molecular identifier; NIPT: Non-invasive prenatal testing; FF: Fetal \\ fraction.
}

\section{Supplementary Information}

The online version contains supplementary material available at https://doi. org/10.1186/s13104-021-05793-4.

Additional file 1: Figure S1. Genomic organization of the RhD-positive allele $\left(R H D^{*} 01\right)$ and three major RhD-negative alleles (RHD*01N.01, $R H D^{*} 01 E L .01$, and $R H D^{*} 01 N .04$ ). The genomic positions of PCR primers targeted for Rhesus boxes (closed arrowheads) and for the exon 9 regions of the RHD and RHCE genes (open arrowheads) are shown for each allele. The nucleotide bases that distinguish the amplicons from the upstream and the downstream Rhesus boxes ( $\mathrm{G}$ at chr1:25,592,628 and $A$ at chr1:25,662,955) are shown. The nucleotide bases that distinguish the amplicons from RHD exon 9 and RHCE exon 9 region (A at chr $1: 25,648,419$ and $\mathrm{A}$ at $\mathrm{chr} 1: 25,648,515$ in the $R H D$ exon 9 region, and $\mathrm{T}$ at $\operatorname{chr} 1: 25,696,992$ and $\mathrm{G}$ at $\mathrm{chr} 1: 25,696,896$ in the RHCE exon 9) are also shown. The red vertical bar shown in the RHD*01EL01 allele represents the $c .1227 A>G$ variation at $c h r 1: 25,648,453$. Figure S2. Error ratio plots for Rhesus boxes (A) and RHD/RHCE exon 9 (B). Error ratios, ratios of the read number containing the bases other than the reference base to the total read number, were calculated using the total numbers for the positionally identical bases between the upstream and downstream at Rhesus box amplicons (for 105 positions) and between RHD exon9 and RHCE exon 9 amplicons (for 147 positions excluding the position of the $c .1227 \mathrm{~A}>\mathrm{G}$ variation at chr1: 25,648,453). The results for twelve each amplicon libraries for Rhesus boxes (A) and RHD/RHCE exon 9 (B) prepared by the one-step PCR protocol (without UMI) (Table S2) were shown. For each nucleotide position, the maximum ratio, the median ratio, and the minimum ratio among twelve libraries are shown in dots (in red, black, and blue, respectively). The gray-shaded regions (nt 1 to 20 and nt 81 to 105 for Rhesus boxes and nt 1 to 22 and nt 120 to 148 for RHD/RHCE exon 9) correspond to PCR primer sequences. Because of the higher error rates consistently observed in the primer regions than in the internal region, the primer regions were excluded for further analyses. The highest error ratio detected in each type of amplicons is indicated by arrow: $0.060 \%$ at nt 37 for Rhesus boxes amplicons and $0.116 \%$ at nt 40 for RHD/RHCD exon 9 amplicons. The median error ratios for the Rhesus box amplicons (nt 21 to 80 ) ranged from $0.00 \%$ to $0.032 \%$ and those for the RHD/RHCE exon 9 amplicons (nt 23 to 119) ranged from $0.00 \%$ to $0.030 \%$.

Additional file 2: Table S1. List of primers. Table S2. Expected and observed ratios of one-step PCR amplicons from the 12 combinations of the 10:1 mixture of genomic DNAs (A and B). Table S3. Expected and observed ratios of UMl-attached amplicons from the 12 combinations of the 10:1 mixture of genomic DNAs ( $A$ and $B$ ).

\section{Acknowledgements}

The authors thank all participants in the study, and also thank Hiromi Kamura and Keisuke Ishiwata for their technical assistance.

\section{Authors' contributions}

$\mathrm{KN}$ conceived the project. $\mathrm{AH}$ and $\mathrm{KN}$ designed the experiments. $\mathrm{HO}-\mathrm{K}$ and $\mathrm{KN}$ designed bioinformatics pipelines. $\mathrm{AH}$ obtained data. $\mathrm{AH}, \mathrm{HO}-\mathrm{K}, \mathrm{OM}$ and $\mathrm{KN}$ analyzed data. AS, KT, KT, AK, AO, AS and HS contributed to the blood materials. $\mathrm{KN}$ and $\mathrm{AH}$ prepared the initial manuscript draft. $\mathrm{KN}$, FT and $\mathrm{KH}$ reviewed and finalized the manuscript. KH and FT supervised the project. All authors read and approved the final manuscript.

\section{Funding}

K. Hata, the National Center for Child Health and Development of Japan (Grant Number 2019A-4); K. Nakabayashi, the National Center for Child Health and Development of Japan (Grant Number 2020B-9), Takeda Science Foundation, Terumo Foundation.

\section{Availability of data and materials}

The sequence read files (fastq.gz) for amplicon libraries (Additional file 2: Tables S2 and S3) and the shell code for the data analysis procedures (Fig. 1K) are available at DRYAD [17].

\section{Declarations}

\section{Ethics approval and consent to participate}

This study was approved by the Research Ethics Committees of the National Center for Child Health and Development (NCCHD) (approval number: 699, 1545), Showa University (approval number: 233), and Jikei University School of Medicine (approval number: 27-059). Genetic counseling was performed prior to sample collection and written informed consent was obtained from all participants.

\section{Consent for publication}

Not applicable.

\section{Competing interests}

Not applicable.

\section{Author details}

${ }^{1}$ Department of Maternal-Fetal Biology, National Research Institute for Child Health and Development, 2-10-1 Okura, Setagaya, Tokyo 157-8535, Japan. ${ }^{2}$ Department of Medical Genetics and Genomics, Kitasato University Graduate School of Medical Sciences, Kanagawa, Japan. ${ }^{3}$ Center for Maternal-Fetal, Neonatal, and Reproductive Medicine, National Center for Child Health and Development, Tokyo, Japan. ${ }^{4}$ Department of Obstetrics and Gynecology, The Jikei University School of Medicine, Tokyo, Japan. ${ }^{5}$ Faculty of Medicine, University of Tsukuba, Ibaraki, Japan. ${ }^{6}$ Department of Obstetrics and Gynecology, Showa University School of Medicine, Tokyo, Japan. ${ }^{7}$ Laboratory of Developmental Genomics, National Research Institute for Child Health and Development, 2-10-1 Okura, Setagaya, Tokyo 157-8535, Japan.

Received: 6 May 2021 Accepted: 17 September 2021 Published online: 26 September 2021

\section{References}

1. de Haas $M$, Thurik FF, van der Ploeg $C P$, Veldhuisen $B$, Hirschberg $H$, et al. Sensitivity of fetal RHD screening for safe guidance oftargeted anti-D immunoglobulin prophylaxis: prospective cohort study of a nationwide programme in the Netherlands. BMJ. 2016;355:15789.

2. Chen J-C, Lin T-M, Chen YL, Wang Y-H, Jin Y-T, Yue C-T. RHD 1227A is an important genetic marker for $\mathrm{RhD}(\mathrm{el})$ individuals. Am J Clin Pathol. 2004;122:193-8.

3. Gassner C, Doescher A, Drnovsek TD, Rozman P, Eicher NI, et al. Presence of RHD in serologically D-, C/E + individuals: a European multicenter study. Transfusion. 2005:45:527-38.

4. Takahashi K, Migita O, Sasaki A, Nasu M, Kawashima A, et al. Amplicon sequencing-based noninvasive fetal genotyping for RHD-positive D antigen-negative alleles. Clin Chem. 2019;65(10):1307-16.

5. Peng Q, Vijaya Satya R, Lewis M, Randad P, Wang Y. Reducing amplification artifacts in high multiplex amplicon sequencing by using molecular barcodes. BMC Genomics. 2015;16(1):589. 
6. Clement K, Farouni R, Bauer DE, Pinello L. AmpUMI: design and analysis of unique molecular identifiers for deep amplicon sequencing. Bioinformatics. 2018;34(13):i202-10.

7. Chen S, Zhou Y, Chen Y, Gu J. fastp: an ultra-fast all-in-one FASTQ preprocessor. Bioinformatics. 2018;34(17)::884-90.

8. MagočT, Salzberg SL. FLASH: fast length adjustment of short reads to improve genome assemblies. Bioinformatics. 2011:27(21):2957-63.

9. Li H, Durbin R. Fast and accurate long-read alignment with BurrowsWheeler transform. Bioinformatics. 2010;26(5):589-95.

10. Li H, Handsaker B, Wysoker A, Fennell T, Ruan J, et al. The sequence alignment/map format and SAMtools. Bioinformatics. 2009;25(16):2078-9.

11. Yang X, Zhou Q, Zhou W, Zhong M, Guo X, et al. A cell-free DNA barcodeenabled single-molecule test for noninvasive prenatal diagnosis of monogenic disorders: application to $\beta$-thalassemia. Adv Sci (Weinh). 2019;6(11):1802332

12. Zhang J, Li J, Saucier JB, Feng Y, Jiang Y, et al. Non-invasive prenatal sequencing for multiple Mendelian monogenic disorders using circulating cell-free fetal DNA. Nat Med. 2019;25(3):439-47.
13. Ma X, Shao $Y$, Tian L, Flasch DA, Mulder HL, et al. Analysis of error profiles in deep next-generation sequencing data. Genome Biol. 2019;20:50.

14. Bianchi DW, Chiu RWK. Sequencing of circulating cell-free DNA during pregnancy. N Engl J Med. 2018;379(5):464-73.

15. Canick JA, Palomaki GE, Kloza EM, Lambert-Messerlian GM, Haddow $J E$. The impact of maternal plasma DNA fetal fraction on next generation sequencing tests for common fetal aneuploidies. Prenat Diagn. 2013;33(7):667-74.

16. Tam JCW, Chan YM, Tsang SY, Yau Cl, Yeung SY, Au KK, Chow CK. Noninvasive prenatal paternity testing by means of SNP-based targeted sequencing. Prenat Diagn. 2020:40(4):497-506.

17. https://datadryad.org/stash/dataset/doi:10.5061/dryad.4tmpg4fb3. Accessed 23 Sept 2021

\section{Publisher's Note}

Springer Nature remains neutral with regard to jurisdictional claims in published maps and institutional affiliations.
Ready to submit your research? Choose BMC and benefit from:

- fast, convenient online submission

- thorough peer review by experienced researchers in your field

- rapid publication on acceptance

- support for research data, including large and complex data types

- gold Open Access which fosters wider collaboration and increased citations

- maximum visibility for your research: over $100 \mathrm{M}$ website views per year

At BMC, research is always in progress.

Learn more biomedcentral.com/submissions 